Marquette University

e-Publications@Marquette

$12-2015$

\title{
A Circle Has No End: Role of Cyclic Topology and Accompanying Structural Reorganization on the Hole Distribution in Cyclic and Linear Poly-p-phenylene Molecular Wires
}

\author{
Marat R. Talipov \\ Marquette University \\ Ramesh Jasti \\ University of Oregon \\ Rajendra Rathore \\ Marquette University
}

Follow this and additional works at: https://epublications.marquette.edu/chem_fac

Part of the Chemistry Commons

\section{Recommended Citation}

Talipov, Marat R.; Jasti, Ramesh; and Rathore, Rajendra, "A Circle Has No End: Role of Cyclic Topology and Accompanying Structural Reorganization on the Hole Distribution in Cyclic and Linear Poly-p-phenylene Molecular Wires" (2015). Chemistry Faculty Research and Publications. 456.

https://epublications.marquette.edu/chem_fac/456 


\title{
A Circle Has No End: Role of Cyclic Topology and Accompanying Structural Reorganization on the Hole Distribution in Cyclic and Linear Poly-p-phenylene Molecular Wires
}

\author{
Marat R. Talipov \\ Department of Chemistry, Marquette University, \\ Milwaukee, WI \\ Ramesh Jasti \\ Department of Chemistry \& Biochemistry and Material Science \\ Institute, University of Oregon, \\ Eugene, OR \\ Rajendra Rathore \\ Department of Chemistry, Marquette University, \\ Milwaukee, WI
}




\section{Abstract}

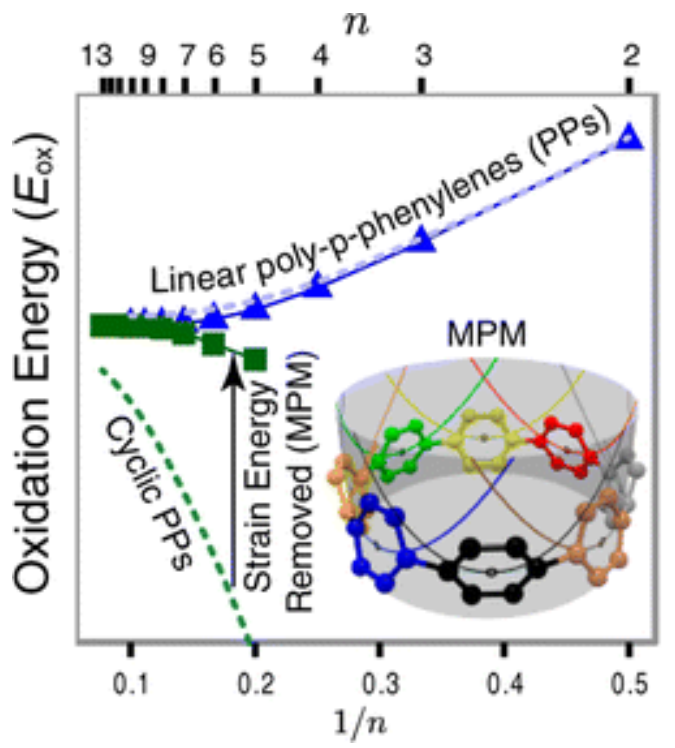

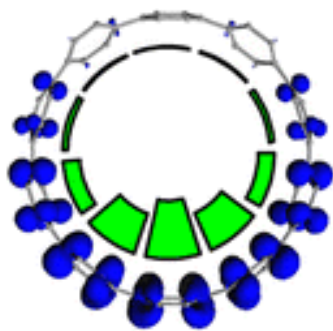

Linear vs Cyclic

Poly-p-phenylenes

\section{Contrasting $E_{\text {ox }}$ trends but Similar Hole Distribution}

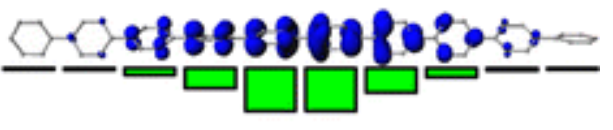

$\Pi$-Conjugated organic oligomers/polymers hold great promise as long-range charge-transfer materials for modern photovoltaic applications. However, a set of criteria for the rational design of functional materials is not yet available, in part because of a lack of understanding of charge distribution in extended $\Pi$-conjugated systems of different topologies, and concomitant effects on redox and optical properties. Herein we demonstrate the role of cyclic versus linear topology in controlling the redox/optical properties and hole distribution in poly- $p$-phenylenes (PPs) with the aid of experiment, computation, and our recently developed multistate parabolic model (MPM). It is unequivocally shown that the hole distribution in both cyclic and linear poly$p$-phenylene $(n \geq 7)$ cation radicals is limited to seven $p$-phenylene units, despite the very different topologies. However, the effect of topology is evidenced in the very different trends in oxidation potentials of cyclic versus linear PPs, which are shown to originate largely from the geometrical distortion of individual $p$-phenylene units in cyclic PPs. The presence of additional pairwise electronic coupling element in cyclic PPs, absent in linear PPs, plays a significant role only in smaller cyclic $\mathrm{PP}_{5}$ and $\mathrm{PP}_{6}$. This study provides a detailed conceptual description of cyclic and linear poly- $p$ phenylene cation radicals and demonstrates the versatility and predictive power of MPM, an important new tool for the design and synthesis of novel and efficient charge-transfer materials for molecular electronics and photovoltaic applications, an area of widespread interest.

\section{Introduction}

A variety of new $n$-conjugated organic polymers with different topologies (e.g., linearly connected poly- $p$-phenylenes, dendritic and 
cyclic structures) have been synthesized with the promise of enhanced electronic and optoelectronic properties for potential applications in the emerging areas of molecular electronics and photovoltaics.(1-3) Unfortunately, a set of well-defined guiding principles has not yet emerged, which could provide a priori knowledge of the dynamics of charge transfer in the polymers of different topologies in order to guide the design and synthesis of efficient long-range charge-transfer materials. In this regard, it is of particular interest to carefully explore both experimentally and computationally the charge distribution in $\Pi$ conjugated polymers of different topologies and its effect on their redox and optical properties.

We recently performed a detailed analysis of the electronic structures of various linear poly- $p$-phenylene cation radicals (CRs) ${ }^{R} \mathbf{P P}_{n}{ }^{*+}$, using a combined computational/experimental approach,(4) and showed that a positive charge (hole) in ${ }^{\boldsymbol{R}} \mathbf{P} \mathbf{P}_{n}{ }^{\cdot+}$ is distributed only over seven monomer units (Figure 1). This confinement of the hole in long poly- $p$-phenylene chains (i.e., $n>7$ ) originates from the interplay between the energetic gain from charge delocalization and concomitant energetic penalty from the structural reorganization (i.e., bond contractions/elongations, angular deformations, and dihedral angles between $p$-phenylenes) as well as the solvent reorganization. We also noted that in the presence of end-capping groups (e.g., alkylor alkoxy-groups) in poly-p-phenylene $\left({ }^{\boldsymbol{R}} \mathbf{P} \mathbf{P}_{n}{ }^{\circ+}\right)$ chains the hole gravitates from the central position to the end of the chain (Figure 1).

Recently, a series of cyclic poly-p-phenylenes ( $\left.{ }^{C} \mathbf{P} \mathbf{P}_{n}\right)$ have been synthesized,(5-11) where the cyclic topology dictates that all of the $p$ phenylene units are identical.(12) This raises the question of whether a hole should be fully delocalized, unlike the linear poly- $p$-phenylenes where the end $p$-phenylene groups are electronically different from the internal phenylene units (Figure 1).(4) This curious notion is further enforced by the reported experimental redox properties of ${ }^{C} \mathbf{P} \mathbf{P}_{n}$ which were starkly different from those of their linear analogues $\left({ }^{\boldsymbol{R}} \mathbf{P} \mathbf{P}_{n}\right)$ (Figure S1 and Tables S1-S4 in the Supporting Information for the compilation of optoelectronic data for ${ }^{C} \mathbf{P} \mathbf{P}_{n} /{ }^{C} \mathbf{P} \mathbf{P}_{n}{ }^{++}$and $\left.{ }^{\boldsymbol{R}} \mathbf{P} \mathbf{P}_{n} /{ }^{\boldsymbol{R}} \mathbf{P} \mathbf{P}_{n}{ }^{-+}\right) \cdot(5-11,13-25)$ 
This difference in the redox and optoelectronic properties of linear and cyclic poly-p-phenylenes (e.g., the oxidation potentials $\left[E_{0 \times 1}\right]$ of linear ${ }^{\boldsymbol{R}} \mathbf{P} \mathbf{P}_{n}$ decrease with increasing number of phenylene units $n$, while $E_{\text {ox } 1}$ of ${ }^{C} \mathbf{P} \mathbf{P}_{n}$ increase with increasing $n$ ) has its origin in part due to the geometry distortion in neutral ${ }^{C} \mathbf{P} \mathbf{P}_{n}$ and the resulting cation radicals. However, it is not clear what role the cyclic topology will play in controlling the extent of the hole distribution in larger cyclic poly- $p$-phenylenes. This is the question we address here, making use of carefully benchmarked DFT calculations and our recently developed multistate parabolic model (MPM)(4) to ascertain the role of topology on the redox and optical properties of linear versus cyclic poly- $p$ phenylenes. To this end, we have extended the MPM to polar coordinates, which allowed an accurate description not only of the hole distribution in ${ }^{C} \mathbf{P} \mathbf{P}_{n}{ }^{++}$but also of the evolution of redox and optical properties.

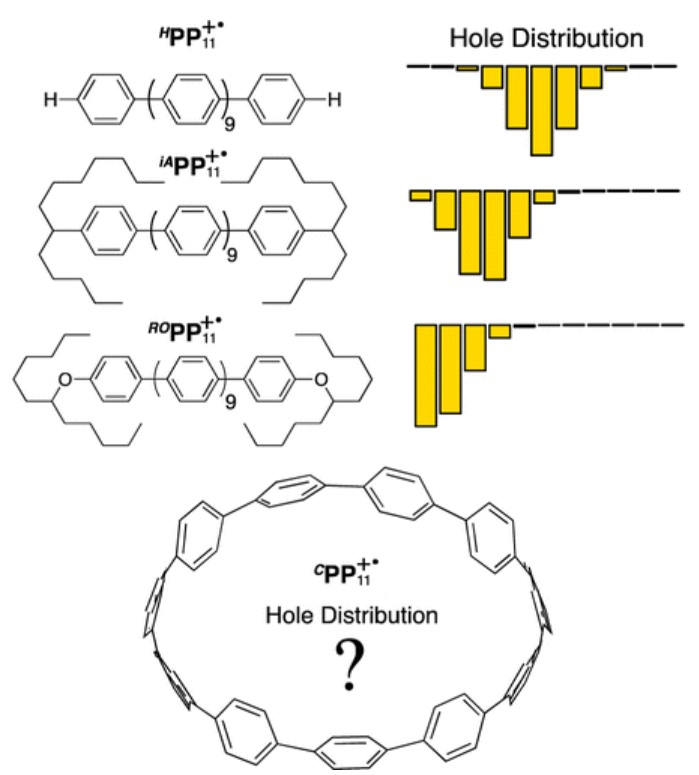

Figure 1. Per-unit hole distribution (represented as bar plots) in the uncapped $\left({ }^{\boldsymbol{H}} \mathbf{P} \mathbf{P}_{11}{ }^{\circ+}\right)$, alkyl-capped $\left({ }^{i \mathbf{A}} \mathbf{P} \mathbf{P}_{11}{ }^{{ }^{++}}\right)$, and alkoxy-capped $\left({ }^{\mathbf{R O}} \mathbf{P} \mathbf{P}_{11}{ }^{\circ+}\right)$ poly- $p$-phenylene cation radicals,, \pm and a representative structure of the cyclic poly-p-phenylene.

\section{Results and Discussion}

\section{DFT Calculations}

Reliable evaluation of the charge/hole delocalization in $n-$ conjugated cation radicals (CRs) is challenging for DFT methods due to 
the self-interaction error;(26-28) however, this problem can be overcome by incorporation of the proper level of Hartree-Fock (HF) exchange.(4, 27, 29-36) Thus, calibration of the one-parameter density functional B1LYP(37) for the ${ }^{\boldsymbol{R}} \mathbf{P P}_{n}{ }^{\bullet+} \operatorname{series}(4,13)(\mathrm{R}=$ isoalkyl, iA; alkoxy, $\boldsymbol{R O}$ groups) resulted in the modified B1LYP-40 functional with $40 \%$ admixture of the HF term [i.e., B1LYP-40/6$31 \mathrm{G}(\mathrm{d})+\mathrm{PCM}\left(\mathrm{CH}_{2} \mathrm{Cl}_{2}\right)$, see the Supporting Information for computational details]. To ensure the applicability of the B1LYP-40 functional for the ${ }^{\mathbf{C}} \mathbf{P} \mathbf{P}_{n}{ }^{\bullet+}$ series, we optimized geometries of ${ }^{C} \mathbf{P} \mathbf{P}_{n} /{ }^{C} \mathbf{P} \mathbf{P}_{n}{ }^{\cdot+}$ for $n=5-13$, and obtained the oxidation energies ( $E^{\mathrm{el}} \mathrm{s}$ ) of ${ }^{C} \mathbf{P} \mathbf{P}_{n}$. The calculated trend of $E^{\mathrm{el}}$ with increasing number of $p$ phenylene units agrees well with the experimentally observed trends both for cyclic and linear poly-p-phenylenes (Figure $\left.2 A, A^{\prime}\right) \cdot(38)$

As a further check of our method, we calculated the energy of the $\mathrm{D}_{0} \rightarrow \mathrm{D}_{1}$ electronic transition in the poly- $p$-phenylene cation radicals (i.e., excitation energies of ${ }^{C} \mathbf{P} \mathbf{P}_{n}{ }^{{ }^{+}}$) using the TD-DFT formalism. The observed trends in the computed excitation energies of ${ }^{C} \mathbf{P P}_{n}{ }^{\bullet+}$ were also in good agreement with the experimental trend (Figure 2B, $\mathrm{B}^{\prime}$ ). Moreover, a comparison of the evolution of the experimental and computed trends for linear ${ }^{\boldsymbol{R}} \mathbf{P} \mathbf{P}_{n}{ }^{{ }^{+}+}$and cyclic ${ }^{\boldsymbol{C}} \mathbf{P} \mathbf{P}_{n}{ }^{\bullet+}$ in Figure 2B, $\mathrm{B}^{\prime}$ showed that both series saturate at $n=8$, and the excitation energy converges at the value of $v_{\max } \sim 5000 \mathrm{~cm}^{-1}$ indicating that the cyclic topology of long ${ }^{\mathbf{C}} \mathbf{P} \mathbf{P}_{n}{ }^{\bullet+}$ plays little role in controlling their optical properties.
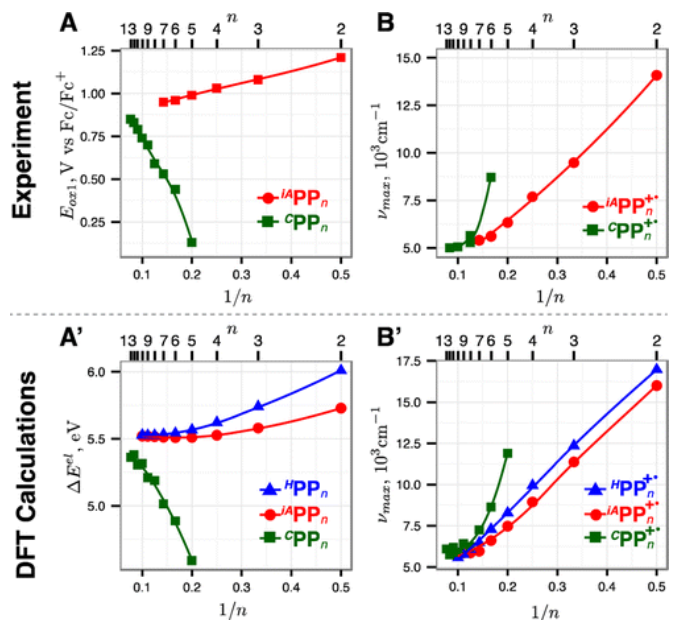

Figure 2. Top: Experimental first oxidation potentials $E_{\text {ox } 1}$ of ${ }^{C} \mathbf{P} \mathbf{P}_{n}$ and ${ }^{i} \mathbf{P} \mathbf{P}_{n}(\mathrm{~A})$, and excitation energies of the corresponding cation radicals (B) vs $1 / n$, where $n$ is the number of $p$-phenylene units. $\frac{39}{}$ Bottom: Calculated (TD-)DFT energies of oxidation of 
various ${ }^{\boldsymbol{R}} \mathbf{P} \mathbf{P}_{n}\left(\mathrm{~A}^{\prime}\right)$, and vertical $\left(\mathrm{D}_{0} \rightarrow \mathrm{D}_{1}\right)$ excitation energies of the ${ }^{\boldsymbol{R}} \mathbf{P} \mathbf{P}_{n}{ }^{\cdot+}\left(\mathrm{B}^{\prime}\right)$ vs $1 / n$ [B1LYP-40/6-31G(d)+PCM $\left.\left(\mathrm{CH}_{2} \mathrm{Cl}_{2}\right)\right]$. Also, see Tables S1-S4 in the Supporting Information for the compilation of the redox/optical properties of ${ }^{C} \mathbf{P P}_{n} \cdot \frac{5-11,13-25}{}$ Note that complete experimental data are not yet available for ${ }^{\mathbf{H}} \mathbf{P P}_{n}$.

Analysis of the electronic structure of various (neutral) poly- $p$ phenylenes showed that the HOMO is fully delocalized in cyclic ${ }^{\mathbf{C}} \mathbf{P} \mathbf{P}_{n}$ while in linear ${ }^{\boldsymbol{R}} \mathbf{P} \mathbf{P}_{n}$ it gravitates toward the center of the molecule (Figure $3 \mathrm{~A}$, see also Figure S4 in the Supporting Information). Thus, it can be expected from the uniform HOMO distribution in ${ }^{\mathbf{C}} \mathbf{P} \mathbf{P}_{n}$ that spin/charge in the corresponding ${ }^{\mathbf{C}} \mathbf{P} \mathbf{P}_{n}{ }^{\bullet+}$ should also be distributed uniformly (see bar plots in Figure $3 \mathrm{~A}$ ). However, this expectation is met only for smaller ${ }^{\boldsymbol{C}} \mathbf{P} \mathbf{P}_{5}{ }^{{ }^{*+}}$ and ${ }^{\boldsymbol{C}} \mathbf{P} \mathbf{P}_{6}{ }^{\bullet+}$, while starting with ${ }^{\boldsymbol{C}} \mathbf{P} \mathbf{P}_{7}{ }^{\bullet+}$, the hole gravitates toward one side of the cycle (Figure $3 \mathrm{~B}$ ). Indeed, in larger ${ }^{C} \mathbf{P P}_{n}{ }^{{ }^{+}}(n \geq 7)$, the hole distribution (Figure $\left.3 \mathrm{~B}\right)$ is clearly confined to no more than seven units, which is uncannily similar to the observed hole distribution over seven units in linear ${ }^{\boldsymbol{R}} \mathbf{P} \mathbf{P}_{n}{ }^{\bullet+}$. The corresponding spin/charge distribution in the excited $\left(D_{1}\right)$ state of various ${ }^{\mathbf{C}} \mathbf{P} \mathbf{P}_{n}{ }^{\bullet+} /{ }^{\mathbf{R}} \mathbf{P} \mathbf{P}_{n}{ }^{\bullet+}$ (Figure $3 \mathrm{C}$ ) again shows a remarkable similarity between the larger $(n>6)$ cyclic and linear poly- $p$-phenylenes. Note that the hole is redistributed in the excited state in such a way that it spreads away from the center leaving the central unit/s virtually unoccupied (Figure $3 \mathrm{C}$ ) which resembles the probability distribution in a simple quantum harmonic oscillator model (Figure S7 in the Supporting Information). Thus, in both ${ }^{\mathbf{C}} \mathbf{P} \mathbf{P}_{n}{ }^{\bullet+}$ and ${ }^{\boldsymbol{R}} \mathbf{P} \mathbf{P}_{n}{ }^{\bullet+}$, the hole in the excited state expands from seven (in the ground state) to eight phenylene units, which also agrees well with the observed saturation of $\mathrm{V}_{\max }$ energies of ${ }^{C} \mathbf{P} \mathbf{P}_{n}{ }^{++} /{ }^{\mathbf{R}} \mathbf{P} \mathbf{P}_{n}{ }^{++}$at $n=8$ (i.e., Figure $2 \mathrm{~B}^{\prime}$ ).

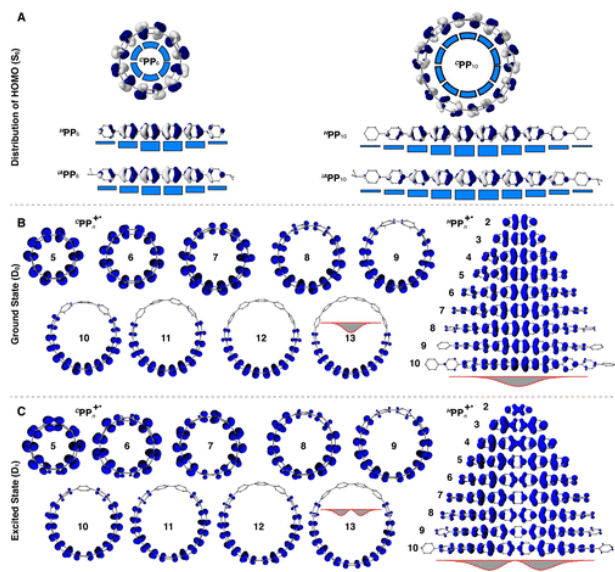

Figure 3. (A) Representative HOMOs $(0.03 \mathrm{au})$ for the cyclic ${ }^{\boldsymbol{C}} \mathbf{P P}_{n}$ and linear ${ }^{H} \mathbf{P P}_{n} /{ }^{i A} \mathbf{P} \mathbf{P}_{n}$ for $n=6$ and 10 and the corresponding HOMO densities [calculated as $q_{m}$

Journal of the American Chemical Society, Vol 137, No. 47 (December 2015): pg. 14999-15006. DOI. This article is (c) American Chemical Society and permission has been granted for this version to appear in e-Publications@Marquette. American Chemical Society does not grant permission for this article to be further copied/distributed or hosted elsewhere without the express permission from American Chemical Society. 
NOT THE PUBLISHED VERSION; this is the author's final, peer-reviewed manuscript. The published version may be accessed by following the link in the citation at the bottom of the page.

$=\Sigma_{n} C_{m n}{ }^{2}$ where $c_{m n}$ is a coefficient of atomic orbital $\mathrm{X}_{m n}$ in HOMO ( is the index of a $p$-phenylene unit, and $n$ is an index of atomic orbital in unit $m$ ] are shown as the bar plots. $(B, C)$ Spin density distribution plots $(0.001 \mathrm{au})$ in the (B)

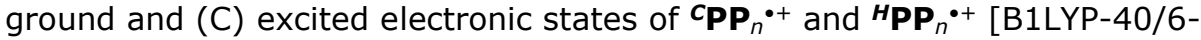

$31 \mathrm{G}(\mathrm{d})+\mathrm{PCM}\left(\mathrm{CH}_{2} \mathrm{Cl}_{2}\right)$ ], which resemble the probability densities of the ground and first state of the quantum harmonic oscillator model. Also, see Figures S6 and S7 in the Supporting Information.

\section{Multistate Model}

To understand the origin of the overall similarity of the hole distribution in the ground $\left(D_{0}\right)$ and excited $\left(D_{1}\right)$ states of cyclic versus linear $\mathbf{P} \mathbf{P}_{n}{ }^{-+}$, which contrast the observed opposite trends in evolution of their oxidation energies (Figure $2 A, A^{\prime}$ ), we make use of the reconfigured multistate parabolic model (MPM). The original MPM, described in detail in a recent article by Rathore and co-workers, $(4)$ uses a quadratic function to represent diabatic free energies of each $p$ phenylene unit. These are treated as identical in the case of parent ${ }^{H} \mathbf{P} \mathbf{P}_{n}{ }^{*+}$ while in the case of end-capped linear ${ }^{\boldsymbol{R}} \mathbf{P} \mathbf{P}_{n}{ }^{-+}$the terminal units were of lower energies as compared to the internal units. The interactions between the $p$-phenylene units (Figure 4A) are modeled using the effective tight-binding Hamiltonian with a constant coupling parameter $H_{a b}$ (eq 1)

$$
\begin{gathered}
\mathbf{H}(x)=\lambda\left[\begin{array}{ccccc}
H_{1}(x) & H_{a b} / \lambda & \cdot & 0 & 0 \\
H_{a b} / \lambda & H_{2}(x) & \cdot & H_{a b} / \lambda & 0 \\
\cdot & \cdot & \cdots & \cdot & \cdot \\
0 & H_{a b} / \lambda & \cdot & H_{n-1}(x) & H_{a b} / \lambda \\
0 & 0 & \cdot & H_{a b} / \lambda & H_{n}(x)
\end{array}\right]_{(1 \mathrm{a})} \\
H_{i}(x)=\left(x-x_{\mathrm{i}}\right)^{2}(1 \mathrm{~b})
\end{gathered}
$$

where $x$ is the combined solvent/geometry reorganization coordinate (i.e., the position of the hole), $x_{i}=$ the position of $i$ th monomer unit, $H_{i}$ = diabatic energy of ith monomer (i.e., energy of the noninteracting monomer unit), $H_{a b}=$ the coupling strength between the neighboring units, and $\lambda=$ the reorganization energy. 


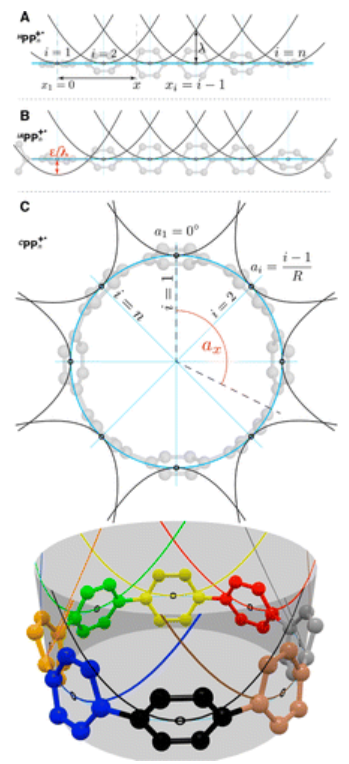

Figure 4. Graphical representation of the multistate parabolic models of (A) ${ }^{\boldsymbol{H}} \mathbf{P P}_{n}{ }^{\bullet+}$, (B) ${ }^{i \boldsymbol{A}} \mathbf{P} \mathbf{P}_{n}{ }^{\bullet+}$, and (C) ${ }^{C} \mathbf{P} \mathbf{P}_{n}{ }^{\bullet+}$.

The eigenvalue and eigenvector matrices of the Hamiltonian matrix in eq 1 can be interpreted as free energies and composition of the ground and excited states of ${ }^{\mathbf{R}} \mathbf{P} \mathbf{P}_{n}{ }^{\bullet+}$, respectively. Thus, diagonalization of the matrix $\mathbf{H}(x)$ for systematically varied $x$ produces a free energy profile of ${ }^{\mathbf{R}} \mathbf{P} \mathbf{P}_{n}{ }^{\bullet+}$ with respect to the combined geometry/solvent reorganization coordinate. Position of the global minimum on this profile could be used for analysis of the redox and optical properties of various ${ }^{\boldsymbol{R}} \mathbf{P} \mathbf{P}_{n}{ }^{\cdot+}$ with regard to such factors as the oligomer length or the nature of the end-capping substituent (which can be simulated by lowering of the corner terms $H_{1}$ and $H_{n}$ by a constant factor $\varepsilon / \lambda$, Figure $4 B$ ). Using MPM with the value of $H_{a b} / \lambda$ combined with $\varepsilon / \lambda=0$ and 3.7 for $\boldsymbol{R}=\boldsymbol{H}$ and $\boldsymbol{i A}$, respectively, reproduced evolution of optoelectronic properties of ${ }^{\boldsymbol{R}} \mathbf{P} \mathbf{P}_{n}{ }^{+\bullet}$ in close agreement with the DFT results and experimental (electrochemistry and absorption spectra) observations.(4)

As "a circle has no beginning or end", employment of the multistate parabolic model, developed for linear poly- $p$-phenylenes, to cyclic ${ }^{\boldsymbol{C}} \mathbf{P} \mathbf{P}_{n}{ }^{\circ+}$ required the following modifications. First, the corner elements of the Hamiltonian in eq $1\left(\mathbf{H}_{1, n}\right.$ and $\left.\mathbf{H}_{n, 1}\right)$ were set to $H_{\mathrm{ab}} / \lambda$ to enable the interaction between the first and last monomer units, which is absent in linear ${ }^{\boldsymbol{R}} \mathbf{P} \mathbf{P}_{n}{ }^{\bullet+}$. Next, the diabatic energies $H_{i}$ were modified to account for the hole delocalization along the circular path of the 
${ }^{C} \mathbf{P} \mathbf{P}_{n}{ }^{\bullet+}$ nanohoop. As the nanohoop radius $(R)$ is constant within a given ${ }^{C} \mathbf{P} \mathbf{P}_{n}{ }^{\bullet+}$, the hole position can be solely determined by the polar angle $a_{x}$ (Figure $\left.4 \mathrm{C}\right)$. The energy of a $p$-phenylene unit $H_{i}$ in the circular path for a given ${ }^{\mathbf{C}} \mathbf{P P}_{n}{ }^{\bullet+}$ can be again represented by a quadratic function

$$
\begin{gathered}
\mathbf{H}\left(a_{x}\right)=\lambda\left[\begin{array}{ccccc}
H_{1}\left(a_{x}\right) & H_{a b} / \lambda & \cdot & 0 & H_{\mathrm{ab}} / \lambda \\
H_{a b} / \lambda & H_{2}\left(a_{x}\right) & \cdot & H_{\mathrm{ab}} / \lambda & 0 \\
\cdot & \cdot & \cdots & \cdot & \cdot \\
0 & H_{a b} / \lambda & \cdot & H_{n-1}\left(a_{x}\right) & H_{\mathrm{ab}} / \lambda \\
H_{a b} / \lambda & 0 & \cdot & H_{\mathrm{ab}} / \lambda & H_{n}\left(a_{x}\right)
\end{array}\right]_{(2 \mathrm{a})} \\
H_{\mathrm{i}}\left(a_{x}\right)=R^{2} \min \left(\left|a_{i}-a_{x}\right|, 2 \pi-\left|a_{i}-a_{x}\right|\right)^{2}(2 \mathrm{~b}) \\
R=\frac{n}{2 \pi} \quad a_{i}=\frac{i-1}{R}(2 \mathrm{c}, \mathrm{d})
\end{gathered}
$$

where $a_{x}$ is the polar angle corresponding to the combined solvent/geometry reorganization (i.e., the position of the hole) and $a_{i}$ $=$ position of $i$ th monomer unit. As the hole delocalizes over no more than seven units in both cyclic ${ }^{C} \mathbf{P} \mathbf{P}_{n}{ }^{\bullet+}$ and linear ${ }^{\mathbf{R}} \mathbf{P} \mathbf{P}_{n}{ }^{\bullet+}$ series (Figure $\underline{3 B}$ ), the value of $H_{\mathrm{ab}} / \lambda=9.0$, used for various ${ }^{\boldsymbol{R}} \mathbf{P} \mathbf{P}_{n}{ }^{\bullet+}$, was also employed for cyclic ${ }^{C} \mathbf{P P}_{n}{ }^{\bullet+}$ (see also Table S5 and its discussion in the Supporting Information).

The models presented in eqs 1 and $\underline{2}$ assume that $H_{i}$ and $H_{\mathrm{ab}} / \lambda$ are independent of the oligomer length of both linear and cyclic poly$p$-phenylenes (i.e., number of $p$-phenylene units). However, the cyclic topology of $\mathbf{C P}_{n} \mathbf{P}^{\cdot+}$ introduces both bending and quinoidal distortion in each $p$-phenylene unit, $(17,19,34,36,40,41)$ the extent of which is dependent on the size of ${ }^{\mathbf{C}} \mathbf{P} \mathbf{P}_{n}{ }^{{ }^{*+}}$. Interestingly, the experimentally observed $(19,40,41)$ quinoidal geometrical distortion in ${ }^{\mathbf{C}} \mathbf{P} \mathbf{P}_{n}$ bears a striking resemblance to the $\mathrm{X}$-ray crystallographically characterized quinoidal distortion of a $p$-phenylene unit in oxidized ${ }^{\boldsymbol{R}} \mathbf{P} \mathbf{P}_{n}{ }^{\cdot+} \cdot(13,42)$ For this reason, oxidation of a $p$-phenylene unit in ${ }^{\mathbf{C}} \mathbf{P} \mathbf{P}_{n}$ should endure significantly reduced (geometrical) reorganization penalty, and therefore, the oxidation energies are expected to lower considerably when compared to the corresponding linear ${ }^{\boldsymbol{R}} \mathbf{P} \mathbf{P}_{n}$ (Figure 5). Thus, the quinoidal distortion of $p$-phenylene units in smaller ${ }^{\boldsymbol{C}} \mathbf{P} \mathbf{P}_{n}{ }^{{ }^{++}}$should decrease the value of $H_{i}\left({ }^{C} \mathbf{P} \mathbf{P}_{n}{ }^{++}\right)$, and therefore, an oligomer size- 
dependent correction factor $D_{n}$ needs to be incorporated into the Hamiltonian from eq 2 , i.e.

$$
H_{i}\left({ }^{C} \mathbf{P p}_{n}^{+\bullet}\right)=H_{i}\left({ }^{C} \mathbf{P P}_{\infty}{ }^{+\bullet}\right)+D_{n} \quad D_{n}<0(3)
$$

where $H_{i}\left({ }^{C} \mathbf{P P}_{\infty}{ }^{\cdot+}\right)$ is identical to $H_{i}$ in eq $2 \mathrm{~b}$ in which the deformation of $p$-phenylene units due to the cyclic topology in ${ }^{C} \mathbf{P} \mathbf{P}_{n}{ }^{++}$was neglected, and is also identical to the $H_{i}$ term for the undistorted linear ${ }^{\boldsymbol{R}} \mathbf{P} \mathbf{P}_{n}{ }^{\cdot+}$ in eq 1.
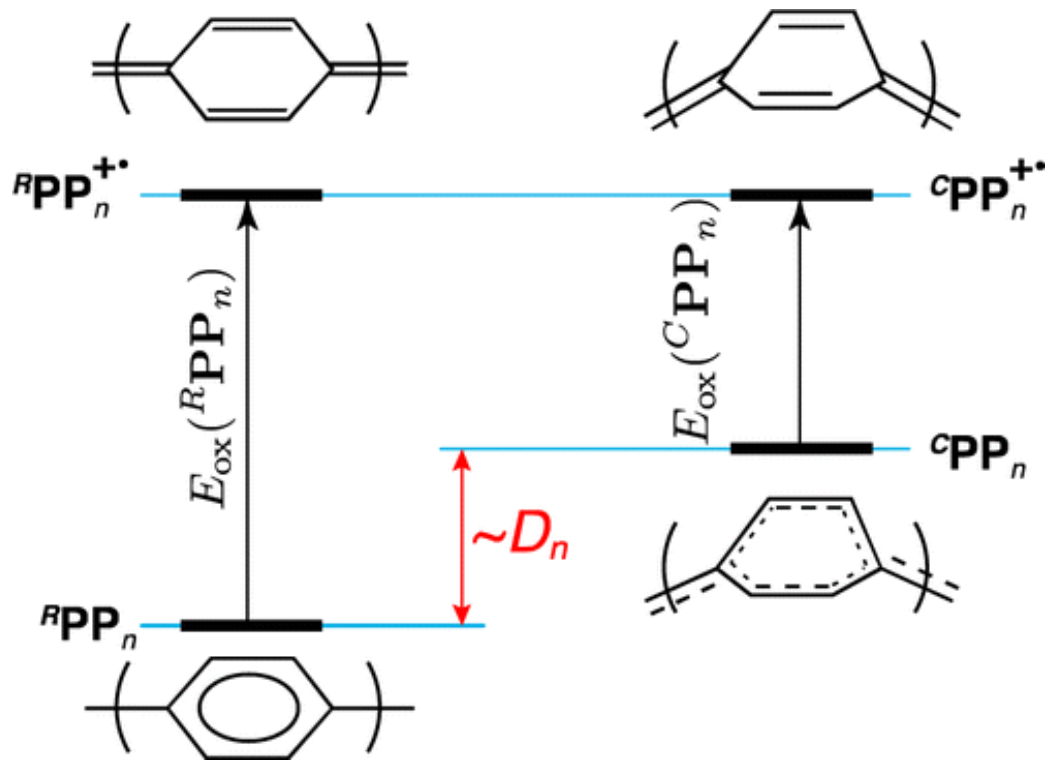

Figure 5. Energetic diagram of the structural changes associated with $1 \mathrm{e}^{-}$oxidation of a single $p$-phenylene unit in linear $\boldsymbol{R} \mathbf{P} \mathbf{P}_{n}{ }^{++}$and cyclic ${ }^{C} \mathbf{P} \mathbf{P}_{n}{ }^{{ }^{+}}$series, and the effect of the enforced geometrical distortions in $\mathbf{C P}_{n}$ due to the cyclic topology.

The distortion factor $D_{n}$ is constant for each $p$-phenylene unit because all $p$-phenylene units are identical in a given cyclic poly- $p$ phenylene, and therefore the model Hamiltonian of ${ }^{C} \mathbf{P} \mathbf{P}_{n}{ }^{\cdot+}$ can be rewritten as

$$
\mathbf{H}_{\text {ext }}\left(a_{x}\right)=D_{n} \mathbf{I}+\mathbf{H}\left(a_{x}\right)(4)
$$

where $\mathbf{I}$ is the $n \times n$ identity matrix, and $\mathbf{H}\left(a_{x}\right)$ is the Hamiltonian from eq $2 \mathrm{a}$.

Diagonalization of the model Hamiltonian matrices $\mathbf{H}_{\text {ext }}(\underline{\text { eq } 4})$ or $\mathbf{H}$ (eq 2a) results in identical eigenvectors (i.e., distribution of hole) 
while the eigenvalues (i.e., free energies) differ by a constant distortion factor $D_{n}$ (see Supporting Information for additional details). Expectedly, the distortion factor $D_{n}$ adds to the free energies of oxidation but has no impact on the hole distribution. Also, the excitation energies of ${ }^{C} \mathbf{P} \mathbf{P}_{n}{ }^{\cdot+}$ are not impacted by $D_{n}$ because it cancels out in the $G_{2}-G_{1}$ term. Accordingly, the hole distribution and the excitation energies of ${ }^{C} \mathbf{P} \mathbf{P}_{n}{ }^{\cdot+}$ can be directly obtained using a simplified Hamiltonian in eq 2 , which does not contain the $D_{n}$ term.

Using the MPM (eq 2), the per-unit hole distribution was calculated for various ${ }^{C} \mathbf{P} \mathbf{P}_{n}{ }^{\cdot+}$ and compared with the corresponding hole distributions from the DFT calculations, shown in Figure $6 \mathrm{~A}$ in the form of bar plots. It is clearly seen that the hole delocalization for $n \geq$ 7 is in remarkable agreement with the DFT results.

The MPM also reproduced the hole distribution in the excited state of ${ }^{\boldsymbol{C}} \mathbf{P} \mathbf{P}_{n}{ }^{{ }^{+}+}$for $n \geq 7$. For smaller ${ }^{\boldsymbol{C}} \mathbf{P} \mathbf{P}_{5}{ }^{{ }^{++}}$and ${ }^{\boldsymbol{C}} \mathbf{P} \mathbf{P}_{6}{ }^{{ }^{++}}$, however, the MPM fails to reproduce the even hole distribution predicted by the DFT predictions. This limitation of the MPM in cases of ${ }^{C} \mathbf{P} \mathbf{P}_{5}{ }^{\bullet+}$ and ${ }^{\mathbf{C}} \mathbf{P} \mathbf{P}_{6}{ }^{{ }^{*+}}$ is not surprising owing to the fact that in smaller ${ }^{\mathbf{C}} \mathbf{P} \mathbf{P}_{n}$ the $p$-phenylene rings are highly distorted (quinoid-like), and the dihedral angles between neighboring $p$-phenylene units are relatively small; therefore, the reorganization term accompanying one-electron oxidation is nearly zero (Figure 5).(43) Indeed, one could reproduce a DFT-like uniform distribution of charge in ${ }^{C} \mathbf{P} \mathbf{P}_{5}{ }^{{ }^{++}}$and ${ }^{\mathbf{C}} \mathbf{P} \mathbf{P}_{6}{ }^{\bullet+}$ with MPM by making use of a value of $\lambda=0$. Moreover, the validity of the application of MPM to poly- $p$-phenylenes is further supported by the fact that it correctly predicts the evolution of the $D_{0} \rightarrow D_{1}$ excitation energies in either linear ${ }^{\boldsymbol{R}} \mathbf{P} \mathbf{P}_{n}{ }^{\bullet+}$ or cyclic ${ }^{\mathbf{C}} \mathbf{P} \mathbf{P}_{n}{ }^{\bullet+}$ (Figure $6 \mathrm{~B}$ vs $\left.\underline{2} \mathrm{~B} / \mathrm{B}^{\prime}\right)$. 
NOT THE PUBLISHED VERSION; this is the author's final, peer-reviewed manuscript. The published version may be accessed by following the link in the citation at the bottom of the page.

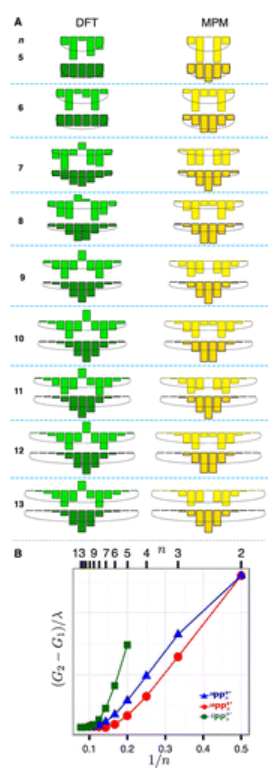

Figure 6. (A) Comparison of the per-unit hole distribution of ${ }^{C} \mathbf{P} \mathbf{P}_{n}{ }^{\cdot+}$ obtained from the DFT calculations (dark/bright green bar plots for the ground/excited states) and multistate parabolic model (dark/bright yellow bar plots for the ground/excited states). The DFT hole distribution was obtained as the per-phenylene natural population analysis charges calculated at the B1LYP-40/6-31G(d)+PCM $\left(\mathrm{CH}_{2} \mathrm{Cl}_{2}\right)$ level, see Figure $\mathrm{S} 8$ in the Supporting Information for additional details. See also Figure S9 in the Supporting Information for a similar plot for various linear ${ }^{\boldsymbol{R}} \mathbf{P P}_{n}{ }^{\cdot+}$.(4) (B) Vertical excitation energies of cyclic ${ }^{C} \mathbf{P} \mathbf{P}_{n}{ }^{\cdot+}$ and linear ${ }^{\boldsymbol{R}} \mathbf{P} \mathbf{P}_{n}{ }^{\cdot+}$, obtained using MPM, vs $1 / n$ (compare with Figure 2B, $\mathrm{B}^{\prime}$ ).

Both MPM and DFT predict remarkably similar hole distribution in cyclic and linear poly- $p$-phenylene cation radicals beyond seven $p$ phenylene units, and the convergence of their $D_{0} \rightarrow D_{1}$ excitation energies, observed both experimentally and computationally, beyond seven $p$-phenylene units, clearly suggests that there is little difference between cyclic and linear topologies as far as hole delocalization/distribution is concerned.(44)

The transformation of a linear poly-p-phenylene into its cyclic

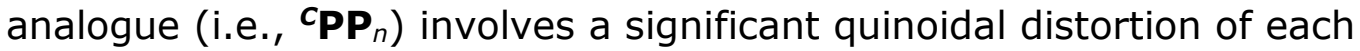
$p$-phenylene ring and an adjustment of dihedral angles between $p$ phenylene units, and the extent of this distortion/structural reorganization decreases with increasing ring size. The consequence of ring size-dependent structural distortion/reorganization is a lowering of the redox potentials of ${ }^{C} \mathbf{P} \mathbf{P}_{n}$ in comparison to the linear poly- $p$ phenylenes. However, one may wonder the following: What is the contribution of the cyclic topology? We can estimate the contribution of cyclic topology in the stabilization of the hole in the ${ }^{C} \mathbf{P} \mathbf{P}_{n}{ }^{\cdot+}$ series by 
discounting the geometrical distortion term $D_{n}$ within the multistate parabolic model, i.e., using the simplified Hamiltonian in eq 2 . The oxidation energies of ${ }^{\mathbf{C}} \mathbf{P} \mathbf{P}_{n}$, evaluated using the modified MPM for cyclic topologies (i.e., eq 2), are plotted in Figure 7 together with the oxidation energies of linear ${ }^{\boldsymbol{R}} \mathbf{P} \mathbf{P}_{n}$ obtained using the original multistate parabolic model (i.e., eq 1). This comparison of the oxidation energies of cyclic and linear poly- $p$-phenylenes in the absence of the distortion term $D_{n}$ showed that the oxidation energies of both cyclic ${ }^{\mathbf{C}} \mathbf{P} \mathbf{P}_{n}$ and linear ${ }^{\boldsymbol{R}} \mathbf{P} \mathbf{P}_{n}$ are the same for $n>7$, which is consistent with the observation of similar hole distribution over seven units in all poly- $p$ phenylenes.

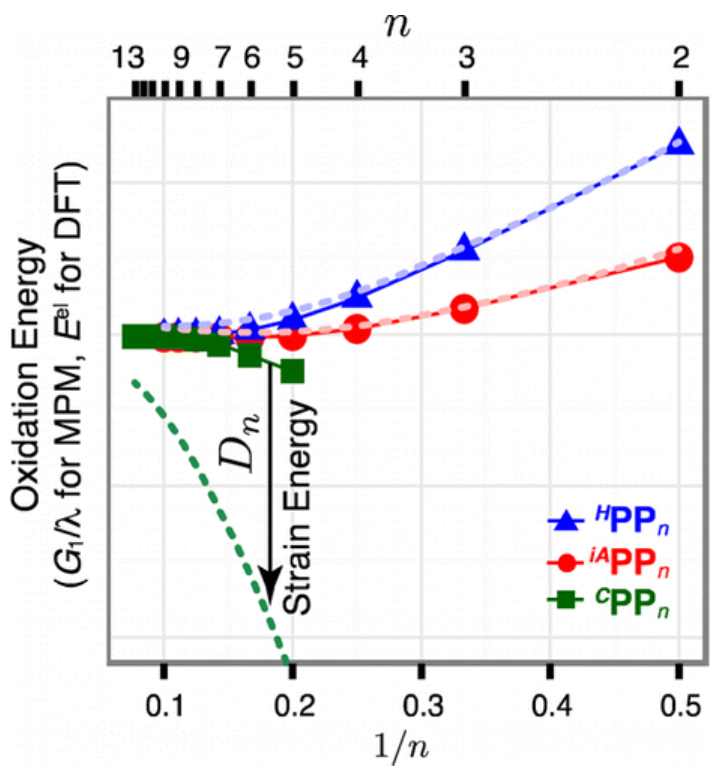

Figure 7. Hole stabilization energies (i.e., oxidation energies) in various $\mathbf{P P}_{n}{ }^{{ }^{+}}$ obtained by MPM (solid lines with symbols). The corresponding dashed lines without symbols show the evolutions of the oxidation energies of various linear and cyclic poly$p$-phenylenes obtained by DFT calculations. Also note the dramatic difference in the evolution of oxidation energies of ${ }^{C} \mathbf{P} \mathbf{P}_{n}$ with and without the distortion factor $D_{n}$.

Interestingly, the smaller ${ }^{\mathbf{C}} \mathbf{P} \mathbf{P}_{n}(n=5-7)$ have somewhat lower oxidation potentials as compared to the corresponding linear analogues (Figure 7). The origin of this lowering of the oxidation potentials in smaller ${ }^{\mathbf{C}} \mathbf{P} \mathbf{P}_{n}$ lies in the fact that cyclic topology introduces an additional pairwise electronic coupling element in the Hamiltonian matrix (eq 2) which is absent in linear ${ }^{\boldsymbol{R}} \mathbf{P P}_{n}$ (eq 1). Thus, the contribution of the additional coupling on the stabilization of the hole (i.e., lowering of the redox potentials) is pertinent only in cases of smaller ${ }^{C} \mathbf{P P}_{n}{ }^{\bullet+}$ because in these ring systems the hole is delocalized 
over all $p$-phenylene units. In large ${ }^{C} \mathbf{P} \mathbf{P}_{n}{ }^{\bullet+}$, the hole distributions are limited to seven $p$-phenylene rings akin to those in linear ${ }^{\boldsymbol{R}} \mathbf{P} \mathbf{P}_{n}{ }^{\bullet+}$, and therefore, an additional coupling element is expected to have no impact on their oxidation energies (Figure 7).

This finding suggests that cyclic topology by virtue of introducing the additional pairwise coupling element plays an important role in stabilizing the cation radicals of smaller ${ }^{\mathbf{C}} \mathbf{P} \mathbf{P}_{n}(n<7)$ while the accompanying quinoidal distortion/reorganization contributes to the stabilization of all ${ }^{\mathbf{C}} \mathbf{P} \mathbf{P}_{n}$ cation radicals, the degree of which decreases with an increase of the size of ${ }^{\mathbf{C}} \mathbf{P} \mathbf{P}_{n}$. Accordingly, one would expect that removal of one $p$-phenylene ring from a larger ${ }^{\boldsymbol{C}} \mathbf{P} \mathbf{P}_{n}$ without altering the remaining structure (i.e., a simple removal of cyclic topology) should not impact its oxidation potential, i.e.

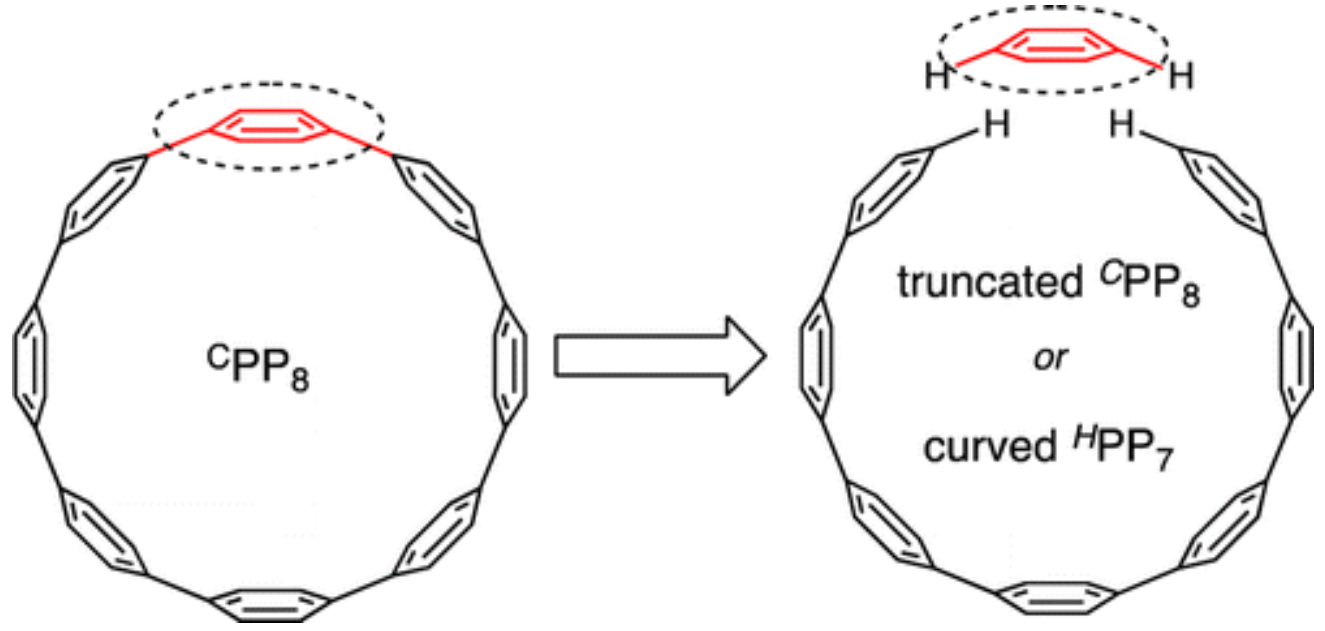

To further verify this finding from MPM analysis and separate the contributions from cyclic topology and accompanying quinoidal distortion/reorganization toward the stabilization of ${ }^{C} \mathbf{P P}_{n}{ }^{\cdot+}$, we performed DFT calculations of these truncated ${ }^{\boldsymbol{C}} \mathbf{P} \mathbf{P}_{n} /{ }^{\boldsymbol{C}} \mathbf{P} \mathbf{P}_{n}{ }^{\bullet+}$, referred to hereafter as curved ${ }^{\boldsymbol{H}} \mathbf{P} \mathbf{P}_{n} /{ }^{\boldsymbol{H}} \mathbf{P} \mathbf{P}_{n}{ }^{\bullet+}$. $(21,45)$ The calculated [B1LYP-40/6$31 \mathrm{G}(\mathrm{d})+\mathrm{PCM}\left(\mathrm{CH}_{2} \mathrm{Cl}_{2}\right)$ ] oxidation energies of curved ${ }^{{ }^{H}} \mathbf{P} \mathbf{P}_{n}$ (see the Supporting Information for details) were compared with the corresponding cyclic ${ }^{\mathbf{P}} \mathbf{P} \mathbf{P}_{n}$ (Figure 8A).

Comparison of the oxidation energies of ${ }^{\mathbf{C}} \mathbf{P} \mathbf{P}_{n}$ and the corresponding curved ${ }^{H} \mathbf{P P}_{n}$ shows identical oxidation potentials for $n>$ 7 , thus suggesting that cyclic topology (i.e., accompanying additional coupling element) does not impact the oxidation energies. However, in 
the case of smaller ${ }^{\boldsymbol{C}} \mathbf{P} \mathbf{P}_{n}$, the effect of cyclic topology is clearly apparent as their oxidation potentials are lower compared to the corresponding curved ${ }^{\mathbf{H}} \mathbf{P} \mathbf{P}_{n}$ (Figure 8A). We also estimated the impact of the quinoidal distortion/reorganization of the $p$-phenylene rings on their oxidation energies using DFT calculations (Figure 8B) which showed that a distorted $p$-phenylene ring from ${ }^{\mathbf{C}} \mathbf{P} \mathbf{P}_{5}$ has a $400 \mathrm{meV}$ lower oxidation energy as compared to that for a distorted $p$ phenylene ring from ${ }^{\mathbf{C}} \mathbf{P} \mathbf{P}_{13 .(46)}$ Moreover, the oxidation energy of the distorted $p$-phenylene ring in ${ }^{C} \mathbf{P} \mathbf{P}_{5}$ is $\sim 500 \mathrm{meV}$ lower than that of the undistorted benzene.

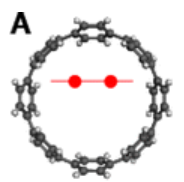

vs

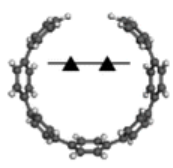

B

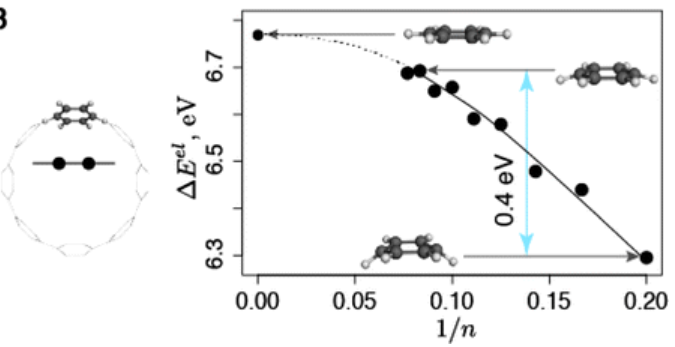

Figure 8. (A) Calculated [B1LYP-40/6-31G(d)+PCM $\left.\left(\mathrm{CH}_{2} \mathrm{Cl}_{2}\right)\right]$ oxidation energies of ${ }^{C} \mathbf{P} \mathbf{P}_{n}$ (red $\bullet$ ) and their truncated analogues (black $\mathbf{\Lambda}$ ) in which one $p$-phenylene unit was removed without altering the remaining structure (missing connections were replaced by the $\mathrm{C}-\mathrm{H}$ bonds, see structures on the left), vs $1 / n$. (B) Calculated oxidation energies of benzene fragment with the distorted geometries as found in various ${ }^{C} \mathbf{P P}_{n}$, vs $1 / n$. Oxidation energy of the undistorted benzene is also included in the plot.

\section{Conclusions}

In this study, we have carefully examined the strikingly different trend with increasing $n$ of experimental $E_{0 \times 1}$ of cyclic ( $\left.{ }^{C} \mathbf{P P}_{n}\right)$ and linear $\left({ }^{R} \mathbf{P} \mathbf{P}_{n}\right)$ poly- $p$-phenylenes and the $\mathrm{D}_{0} \rightarrow \mathrm{D}_{1}$ transition $\left(\mathrm{V}_{\max }\right)$ in their cation radicals using DFT calculations and our reconfigured multistate parabolic model (MPM). Calculations using a modified DFT functional [B1LYP-40/6-31G(d)+PCM $\left(\mathrm{CH}_{2} \mathrm{Cl}_{2}\right)$ ] accurately reproduce the evolution 
of $E_{0 \times 1}$ of cyclic/linear $\mathbf{P} \mathbf{P}_{n}$ as well as the evolution of $\mathbf{v}_{\max }$ in their cation radicals (Figure 2 ). In contrast to linear ${ }^{\boldsymbol{R}} \mathbf{P} \mathbf{P}_{n}$, where the HOMO gravitates toward the center of oligomer chain, in ${ }^{\mathbf{C}} \mathbf{P} \mathbf{P}_{n}$ it is fully delocalized due to the cyclic topology (Figure 3A). The hole (i.e., spin/charge) distribution in smaller ${ }^{\mathbf{C}} \mathbf{P P}_{n}{ }^{\bullet+}(n<7)$ is also fully delocalized, but gravitates toward one side of the ring in larger ${ }^{C} \mathbf{P P}_{n}{ }^{{ }^{++}}$ $(n \geq 7)$ similar to the corresponding linear ${ }^{\mathbf{R}} \mathbf{P} \mathbf{P}_{n}{ }^{\bullet+}$. Importantly, in both series, the hole distribution is limited to only seven $p$-phenylene units (Figure $3 \mathrm{~B}$ ). The similarity of the hole distribution in larger cyclic/linear $\mathbf{P P}_{n}{ }^{\cdot+}$, both in the ground $\left(D_{0}\right)$ and excited $\left(D_{1}\right)$ states, is consistent with the observed convergence of their experimental/calculated $v_{\max }\left(D_{0} \rightarrow D_{1}\right)$ energies at $n>7$ (Figure 2B, $\left.B^{\prime}\right)$.

We have modified our recently developed multistate parabolic model, which fully reconciles the experimental/DFT observables ( $E_{\text {ox1 }}$, $\mathrm{V}_{\text {max }}$ ) for linear ${ }^{\boldsymbol{R}} \mathbf{P} \mathbf{P}_{n} /{ }^{\boldsymbol{R}} \mathbf{P} \mathbf{P}_{n}{ }^{\bullet+}$, by introducing a polar coordinate system to accommodate the cyclic topology of ${ }^{\boldsymbol{C}} \mathbf{P} \mathbf{P}_{n} /{ }^{\mathbf{C}} \mathbf{P} \mathbf{P}_{n}{ }^{\cdot+}$ (Figure 4 ). The modified MPM performed extremely well for larger ${ }^{C} \mathbf{P P}_{n}{ }^{+}, n \geq 7$; however, for smaller ${ }^{\mathbf{C}} \mathbf{P} \mathbf{P}_{5}{ }^{{ }^{++}}$and ${ }^{C} \mathbf{P} \mathbf{P}_{6}{ }^{\bullet+}$ it produced a hole distribution which was not evenly delocalized (Figure 6A). This discrepancy arises largely due to the (severe) pre-existing quinoidal distortion (structural reorganization) of $p$-phenylene units in neutral ${ }^{\mathbf{C}} \mathbf{P P}_{5}$ and ${ }^{\mathbf{C}} \mathbf{P} \mathbf{P}_{6}$ (Figure 5). Despite this limitation, the modified MPM accurately reproduced observed convergence of the $\mathrm{V}_{\max }\left(D_{0} \rightarrow D_{1}\right)$ energies for both cyclic ${ }^{\mathbf{C}} \mathbf{P} \mathbf{P}_{n}{ }^{\bullet+}$ or linear ${ }^{\boldsymbol{R}} \mathbf{P} \mathbf{P}_{n}{ }^{\bullet+}$.

Our study reconciles the strikingly different trend observed in oxidation potential $E_{\text {ox } 1}$, with increasing $n$, which increases for cyclic ${ }^{C} \mathbf{P} \mathbf{P}_{n}$ but decreases for linear ${ }^{\boldsymbol{R}} \mathbf{P} \mathbf{P}_{n}$, and leads to $E_{\text {ox } 1}$ values for a given $n$ that are considerably lower for cyclic ${ }^{\boldsymbol{C}} \mathbf{P P}_{n}$ (Table S3 in the Supporting Information). We show that these trends originate, in part, due to the cyclic topology which (a) imparts severe distortions and lowers the dihedral angles between $p$-phenylene units, and (b) introduces an additional pairwise electronic coupling element (eq 2) which is absent in linear ${ }^{\boldsymbol{R}} \mathbf{P} \mathbf{P}_{n}$. While both points $\mathrm{a}$ and $\mathrm{b}$ are important for smaller ${ }^{C} \mathbf{P P}_{n}(n<7)$, only point a plays a role in lowering the redox potentials of larger ${ }^{\mathbf{C}} \mathbf{P} \mathbf{P}_{n}$ in comparison with those of the linear analogues. 
Most importantly, our combined experimental and DFT/MPM study clearly demonstrates that hole distribution in both linear and cyclic poly- $p$-phenylene cation radicals is strikingly similar in larger $\mathbf{P P}_{n}{ }^{\cdot+}(n \geq 7)$ and is limited to only seven $p$-phenylene units.

Confinement of the hole is shown to arise from the interplay between the energetic gain from electronic coupling/charge delocalization and concomitant energetic penalty from the structural/solvent reorganization, often referred to simply as reorganization energy. In view of its demonstrated versatility and ready applicability, the MPM is shown to be an important new tool for designing novel long-range charge-transport materials for molecular electronics and photovoltaic applications.

\section{Supporting Information}

The Supporting Information is available free of charge on the ACS Publications website at DOI: 10.1021/jacs.5b09596.

- Computational details, Tables S1-S5, Figures S1-S11, and the coordinates and energies of the calculated structures (PDF)

The authors declare no competing financial interest.

\section{Acknowledgment}

We thank the NSF (CHE-1508677) and NIH (R01-HL112639-04) for financial support and Professors S. A. Reid and Q. K. Timerghazin for helpful discussions. R.J. thanks the Camille and Henry Dreyfus Foundation for financial support. The calculations were performed on the high-performance computing cluster Père at Marquette University funded by NSF Awards OCI0923037 and CBET-0521602, and the Extreme Science and Engineering Discovery Environment (XSEDE) funded by the NSF (TG-CHE130101).

\section{References}

1 Li, C.; Liu, M.; Pschirer, N. G.; Baumgarten, M.; Müllen, K. Chem. Rev. 2010, 110, 6817, DOI: $10.1021 /$ cr100052z

${ }^{2}$ Facchetti, A. Chem. Mater. 2011, 23, 733, DOI: 10.1021/cm102419z

sGGuo, X.; Baumgarten, M.; Müllen, K. Prog. Polym. Sci. 2013, 38, 1832, DOI:

10.1016/j.progpolymsci.2013.09.005

4Talipov, M. R.; Boddeda, A.; Timerghazin, Q. K.; Rathore, R. J. Phys. Chem.

C 2014, 118, 21400, DOI: 10.1021/jp5082752

Journal of the American Chemical Society, Vol 137, No. 47 (December 2015): pg. 14999-15006. DOI. This article is (C) American Chemical Society and permission has been granted for this version to appear in e-Publications@Marquette. American Chemical Society does not grant permission for this article to be further copied/distributed or hosted elsewhere without the express permission from American Chemical Society. 
NOT THE PUBLISHED VERSION; this is the author's final, peer-reviewed manuscript. The published version may be accessed by following the link in the citation at the bottom of the page.

5]asti, R.; Bhattacharjee, J.; Neaton, J. B.; Bertozzi, C. R. J. Am. Chem. Soc. 2008, 130, 17646, DOI: 10.1021/ja807126u

6yamago, S.; Watanabe, Y.; Iwamoto, T. Angew. Chem., Int. Ed. 2010, 49, 757, DOI: 10.1002 /anie. 200905659

IIwamoto, T.; Watanabe, Y.; Sakamoto, Y.; Suzuki, T.; Yamago, S. J. Am. Chem. Soc. 2011, 133, 8354, DOI: 10.1021/ja2020668

8Hirst, E. S.; Jasti, R. J. Org. Chem. 2012, 77, 10473, DOI: 10.1021/jo302186h

9Segawa, Y.; Fukazawa, A.; Matsuura, S.; Omachi, H.; Yamaguchi, S.; Irle, S.; Itami, K. Org. Biomol. Chem. 2012, 10, 5979, DOI: 10.1039/c2ob25199j

${ }^{10}$ Evans, P. J.; Darzi, E. R.; Jasti, R. Nat. Chem. 2014, 6, 404, DOI: 10.1038/nchem. 1888

11Yamago, S.; Kayahara, E.; Iwamoto, T. Chem. Rec. 2014, 14, 84, DOI: 10.1002/tcr.201300035

${ }^{12}$ Whereas this statement is strictly true for the even-numbered ${ }^{C} \mathbf{P} \mathbf{P}_{n}$, the odd-numbered ${ }^{\boldsymbol{C}} \mathbf{P} \mathbf{P}_{n}$ possess a natural geometrical defect arising from the twist between adjacent $p$-phenylene rings (see, e.g., ref $\underline{36}$ ), which causes a slight dissimilarity amongst the $p$-phenylene units.

13Banerjee, M.; Shukla, R.; Rathore, R. J. Am. Chem. Soc. 2009, 131, 1780, DOI: $10.1021 / j a 805102 d$

14Sisto, T. J.; Golder, M. R.; Hirst, E. S.; Jasti, R. J. Am. Chem. Soc. 2011, 133, 15800, DOI: 10.1021/ja205606p

15 Fujitsuka, M.; Cho, D. W.; Iwamoto, T.; Yamago, S.; Majima, T. Phys. Chem. Chem. Phys. 2012, 14, 14585, DOI: 10.1039/c2cp42712e

16 Xia, J.; Jasti, R. Angew. Chem., Int. Ed. 2012, 51, 2474, DOI: 10.1002/anie.201108167

17Camacho, C.; Niehaus, T. A.; Itami, K.; Irle, S. Chem. Sci. 2013, 4, 187, DOI: $10.1039 / C 2 S C 20878 D$

${ }^{18}$ Golder, M. R.; Wong, B. M.; Jasti, R. Chem. Sci. 2013, 4, 4285, DOI: $10.1039 / \mathrm{c} 3 s c 51861 \mathrm{~b}$

19 Kayahara, E.; Kouyama, T.; Kato, T.; Takaya, H.; Yasuda, N.; Yamago, S. Angew. Chem., Int. Ed. 2013, 52, 13722, DOI: 10.1002/anie. 201306881 Note that the crystal structure of ${ }^{\mathbf{C}} \mathbf{P} \mathbf{P}_{8}{ }^{+2}$ reported in this article is heavily disordered and therefore the subtle bond length changes to discern the extent of distribution of cationic charges is unreliable. Also note the EPR data provided a dynamically-averaged distribution of the hole and cannot necessarily be related to the extent of hole distribution in ${ }^{\mathbf{C}} \mathbf{P P}_{8}{ }^{{ }^{++}}$

20Kayahara, E.; Iwamoto, T.; Suzuki, T.; Yamago, S. Chem. Lett. 2013, 42, 621, DOI: $10.1246 / \mathrm{cl} .130188$ 
NOT THE PUBLISHED VERSION; this is the author's final, peer-reviewed manuscript. The published version may be accessed by following the link in the citation at the bottom of the page.

211Li, P.; Sisto, T. J.; Darzi, E. R.; Jasti, R. Org. Lett. 2014, 16, 182, DOI: $10.1021 /$ ol403168x

222Fujitsuka, M.; Tojo, S.; Iwamoto, T.; Kayahara, E.; Yamago, S.; Majima, T. J. Phys. Chem. Lett. 2014, 5, 2302, DOI: 10.1021/jz5009054 The spectra of ${ }^{\boldsymbol{C}} \mathbf{P} \mathbf{P}_{10}$ and ${ }^{\boldsymbol{C}} \mathbf{P} \mathbf{P}_{12}$ cation radicals reported in this reference were not recorded to necessary spectral width to clearly determine the absorption maxima. Moreover, in the case of ${ }^{C} \mathbf{P} \mathbf{P}_{10^{\circ}}{ }^{\bullet}$, the noise in the spectrum was taken as the absorption maximum, and therefore, the conclusions drawn in this reference are questionable.

233amago, S.; Kayahara, E.; Iwamoto, T. Chem. Rec. 2014, 14, 84, DOI: $10.1002 /$ tcr. 201300035

24Hines, D. A.; Darzi, E. R.; Jasti, R.; Kamat, P. V. J. Phys. Chem. A 2014, 118, 1595, DOI: $10.1021 /$ jp4123562

${ }_{25}^{25}$ Toriumi, N.; Muranaka, A.; Kayahara, E.; Yamago, S.; Uchiyama, M. J. Am. Chem. Soc. 2015, 137, 82, DOI: 10.1021/ja511320f

${ }^{26}$ Cohen, A. J.; Mori-Sánchez, P.; Yang, W. Science 2008, 321, 792, DOI: $10.1126 /$ science. 1158722

27Renz, M.; Theilacker, K.; Lambert, C.; Kaupp, M. J. Am. Chem. Soc. 2009, 131, 16292, DOI: 10.1021/ja9070859.

Compare with refs 22 and 25 .

${ }^{28}$ Cohen, A. J.; Mori-Sánchez, P.; Yang, W. Chem. Rev. 2012, 112, 289, DOI: $10.1021 / \mathrm{cr} 200107 z$

${ }^{29}$ Renz, M.; Kess, M.; Diedenhofen, M.; Klamt, A.; Kaupp, M. J. Chem. Theory Comput. 2012, 8, 4189, DOI: 10.1021/ct300545x

30 Talipov, M. R.; Boddeda, A.; Lindeman, S. V.; Rathore, R. J. Phys. Chem. Lett. 2015, 6, 3373, DOI: 10.1021/acs.jpclett.5b01532

31 Talipov, M. R.; Navale, T. S.; Rathore, R. Angew. Chem., Int. Ed. 2015, available online Oct 1 2015, DOI:, DOI: 10.1002/anie.201506402 .

32 Kilina, S.; Kilin, D.; Tretiak, S. Chem. Rev. 2015, 115, 5929, DOI: 10.1021/acs.chemrev.5b00012

33 Bravaya, K. B.; Epifanovsky, E.; Krylov, A. I. J. Phys. Chem. Lett. 2012, 3, 2726, DOI: $10.1021 / \mathrm{jz} 3011139$

${ }^{34}$ Adamska, L.; Nayyar, I.; Chen, H.; Swan, A. K.; Oldani, N.; FernandezAlberti, S.; Golder, M. R.; Jasti, R.; Doorn, S. K.; Tretiak, S. Nano Lett. 2014, 14, 6539, DOI: 10.1021/nl503133e

35 Parthey, M.; Kaupp, M. Chem. Soc. Rev. 2014, 43, 5067, DOI: $10.1039 / \mathrm{c} 3 \mathrm{cs} 60481 \mathrm{k}$

36Liu, J.; Adamska, L.; Doorn, S. K.; Tretiak, S. Phys. Chem. Chem. Phys. 2015, 17, 14613, DOI: 10.1039/C5CP01782C

${ }^{37}$ Adamo, C.; Barone, V. Chem. Phys. Lett. 1997, 274, 242, DOI: 10.1016/S0009-2614(97)00651-9

${ }^{38}$ DFT calculations predict that odd- and even-numbered ${ }^{\mathbf{C}} \mathbf{P} \mathbf{P}_{n}$ have slightly different $E^{\mathrm{el}}$ trends, in agreement with the evolution of HOMO energies 
NOT THE PUBLISHED VERSION; this is the author's final, peer-reviewed manuscript. The published version may be accessed by following the link in the citation at the bottom of the page.

(see Figure $\mathrm{S} 3$ in the Supporting Information); however, this effect is not realized in experimental studies due to the dynamic averaging, see ref $\underline{7}$.

39The $1 / n$ coordinate is used in this study because redox and optical properties of oligomers, and their cation radicals, can often be approximately linearized against $1 / n$ in the cases of small $n$. Note however that more accurate linearization up to the polymeric limit can be achieved by using the $\cos [n /(n+1)]$ coordinate, see ref $\underline{4}$ and Torras, J.; Casanovas, J.; Alemán, C. J. Phys. Chem. A 2012, 116, 7571, DOI: 10.1021/jp303584b

40Darzi, E. R.; Jasti, R. Chem. Soc. Rev. 2015, 44, 6401, DOI: 10.1039/C5CS00143A

41Fujitsuka, M.; Iwamoto, T.; Kayahara, E.; Yamago, S.; Majima, T. ChemPhysChem 2013, 14, 1570, DOI: 10.1002/cphc.201300144

42Banerjee, M.; Lindeman, S. V.; Rathore, R. J. Am. Chem. Soc. 2007, 129, 8070, DOI: $10.1021 /$ ja0720356

43 Indeed, the oxidation-induced distortion of $p$-phenylene units decreases with decreasing number of $p$-phenylene units in ${ }^{\mathbf{C}} \mathbf{P} \mathbf{P}_{n}$; e.g., the average bond contraction in ${ }^{\mathbf{C}} \mathbf{P} \mathbf{P}_{10}$ is $0.008 \AA$, which is much smaller in the case of ${ }^{C} \mathbf{P P}_{5}(0.005 \AA)$. Moreover, reduced dihedral angles between $p$-phenylenes in ${ }^{\mathbf{C}} \mathbf{P P}_{5}$ and ${ }^{\mathbf{C}} \mathbf{P} \mathbf{P}_{6}$ is expected to increase the interchromophoric coupling, and therefore, it may also contribute to more uniform hole distribution in smaller ${ }^{\boldsymbol{C}} \mathbf{P} \mathbf{P}_{n}$ cation radicals.

${ }^{44}$ Note that cyclic ${ }^{C} \mathbf{P P}_{n}{ }^{{ }^{+}+}$have shallow free energy profiles, which may facilitate the dynamics of the hole along a circular path, but in any given (frozen) electronic structure, the hole distribution will remain limited to seven $p$-phenylene units. See Figure S10 and related discussion in the Supporting Information.

${ }^{45}$ The effect of the cyclic topology was also investigated by the group of Jasti et al. (ref $\underline{21}$ ) by inserting a methylene group between two $p$ phenylenes in ${ }^{\boldsymbol{C}} \mathbf{P} \mathbf{P}_{7}$. See Figure $\mathrm{S} 11$ and related discussion in the Supporting Information.

46 The obtained value of $400 \mathrm{mV}$ is only the lower estimate of the geometrical distortion effect since it neglects distortions that cannot be attributed to one phenylene unit, e.g., the distortion of the $\mathrm{C}-\mathrm{C}$ bond between adjacent $p$-phenylene units. 